\title{
The Effect of Polarization on the Retardation of Non-Relativistic Charged Particles
}

\author{
GUNNAR ANIANSSON \\ Division of Physical Chemistry, Royal Institute of Technology, Stockholm 70, Sweden \\ Dedicated to Professor Ole Lamm on his 60th birthday

\begin{abstract}
The theory for the effect of dielectric polarization on the retardation of non-relativistic charged particles is re-examined. An expression for the effect is obtained in which the dominant term is similar to that obtained by Fermi ${ }^{13}$. It differs by a multiplicative factor and an additive term, both dependent on a parameter describing the form of the oscillator strength distribution.

The influence of the local field correction for the dielectric constant of the medium is also taken into account.
\end{abstract}

\section{INTRODUCTION}

The theory of the retardation of charged particles through loss of energy to 1 the atoms or molecules of the medium traversed was first treated on the assumption that the interaction between the particle and each one of the atoms or molecules could be considered separately ${ }^{1-10}$. It was then remarked by Swann ${ }^{11}$ that molecules, distant from the path of the particle, should be partly shielded from the particle by the dielectric polarization in the intervening molecules. A theory for this phenomenon was worked out by Fermi ${ }^{12,13}$ using methods akin to those used by Frank and Tamm in the theory for the Cerenkov effect. A reduction in the energy loss was predicted which for particles with velocities smaller than the phase velocity of light in the medium was of the order of several percent. For higher velocities the effect eventually becomes dominant. In this theory it was assumed that the dielectric properties of the medium could be adequately described by a single dispersion frequency. This restriction has subsequently been removed and the theory developed for detailed application to particular cases ${ }^{14-25}$. In connection with its application to the retardation of $\alpha$-particles in organic liquids, it was found to contain ambiguities affecting the order of magnitude of the predicted polarization effect ${ }^{26}$. A re-treatment of the theory was therefore undertaken with particular regard to its ultimate application to non-polar molecular liquids.

Acta Chem. Scand. 16 (1962) No. 9 


\section{MICROSCOPIC AND MACROSCOPIC TREATMENT}

In the full quantum-mechanical treatment of the interaction between a fast charged particle and a single molecule ${ }^{8,9}$, the incoming particle is treated as a plane wave. The rate of change of the combined wave function for the particle and the molecule is then expanded into outgoing plane waves of the particle in different directions and with varying wave number combined with the different states of the molecule. The square of the coefficients of the expansion measures the probabilities of the changes of momentum and energy of the particle accompanied by changes in the state of the molecule. In order to obtain the mean retardation of the particle, a sum over the different probabilities has to be performed.

It was, however, proved by Mott ${ }^{27}$ that when the change in momentum experienced by the particle is small its effect on the molecule is that of a classical point charge travelling with constant velocity in a straight line past (or through) the molecule. This situation obtains in particular for heavy particles, such as protons and $\alpha$-particles, except for the rare, large angle deflections against the nuclei of the molecule.

When the velocity, $v$, of the particle is much larger than the average velocity $v_{\mathrm{o}}$ of the electrons in the molecule, it is possible to divide the collisions into two groups ${ }^{3,9}$ according to whether the perpendicular distance between the particle track and the center of the molecule is smaller or larger than a distance $b$ such that

$$
\bar{r}<<b<<\frac{v}{\omega_{\mathrm{o}}}
$$

where $\bar{r}$ is the average distance of the electrons from the center of the molecule $\hbar \omega_{\mathrm{o}}$ is of the order of the electronic excitation energies. In the former case, i.e. close collisions, the electrons may be considered free since the major part of the disturbance caused by the particle is so rapid that the intra-molecular forces have no time to act. In the latter case the electric field from the particle is practically uniform over the whole molecule so that the treatment becomes a pure dispersion problem.

The energy transferred to the molecules of a gas from a traversing particle is now obtainable on the assumption that the disturbance caused in one molecule is not affected by the disturbances set up in the surrounding molecules. The rate of change of the energy $E$ of the particle due to the molecules within the distance $b$ from the particle track becomes ${ }^{9}$

$$
\left(-\frac{\mathrm{d} E}{\mathrm{~d} x}\right)_{<b}=\frac{4 \pi q^{2} e^{4}}{m v^{2}} N Z \ln \frac{2 m v b}{\hbar k}
$$

Here $x$ is the distance along the particle track, $-e$ and $m$ are the charge and mass of the electron, $q e$ and $v$ the charge and velocity of the particle, $N$ the number of molecules per unit volume, $Z$ the number of electrons per molecule, $2 \pi \hbar$ Planck's constant and $k$ a numerical constant $=1.123$. Similarly for the molecules outside the distance $b$ 


$$
\left(-\frac{\mathrm{d} E}{\mathrm{~d} x}\right)_{>b}=\frac{4 \pi q^{2} e^{4}}{m v^{2}} N Z \sum_{i} f_{i} \ln \frac{k v}{\omega_{i}}
$$

where $\hbar \cdot \omega_{i}$ is the energy difference between the $i$ :th excited electronic state and the ground state of the molecules and $f_{i}$ the corresponding oscillator strengths satisfying $\sum_{i} f_{i}=1$.

The total rate of energy loss is then

$$
-\frac{\mathrm{d} E}{\mathrm{~d} x}=\frac{4 \pi q^{2} e^{4}}{m v^{2}} N Z \sum_{i} f_{i} \ln \frac{2 m v^{2}}{\hbar \omega_{i}}
$$

Due to the circumstance that the action of the particle on the molecules outside the radius $b$ from the track is essentially a classical dispersion problem and because the radius $b$ is larger than molecular dimensions, the macroscopic electrodynamic theory should be applicable to this part of the energy loss ${ }^{12,13}$. The description of the dielectric properties of the medium with a continuum theory automatically takes collective effects into account. For the close collisions on the other hand, collective effects should be relatively much smaller, thus allowing the results above for $(-\mathrm{d} E / \mathrm{d} x)_{<b}$ to be employed.

The rate of energy transferred to the medium outside the radius $b$ around the track is determined by the total flux of the Poynting vector out of the cylinder with radius $b$. The energy loss per unit path is then $1 / v$ of this rate:

$$
\left(-\frac{\mathrm{d} E}{\mathrm{~d} x}\right)_{b>}=\frac{c}{4 \pi v} \int(\vec{E} \times \vec{H}) \cdot \mathrm{d} \vec{s}
$$

By Fourier expansion of the field vectors and introducing the complex dielectric constant $\varepsilon(\omega)$, Fermi obtained ${ }^{13,14}$

$$
\left(-\frac{\mathrm{d} E}{\mathrm{~d} x}\right)_{b>}=\frac{i b q^{2} e^{2}}{\pi v^{3}} \int_{\mathrm{o}}^{\infty} \frac{\omega^{2}}{\varepsilon \vartheta^{2} \vartheta^{*}} K_{\mathrm{o}}\left(\frac{\omega b}{\vartheta v}\right) K_{\mathrm{o}}^{\prime}\left(\frac{\omega b}{\vartheta^{*} v}\right) \mathrm{d} \omega
$$

where $1 / \vartheta^{2}=1-\left(v^{2} / c^{2}\right) \varepsilon$.

$$
+ \text { complex conjugate, }
$$

The Bessel function $K_{\mathrm{o}}$ takes a simple form when $|\omega b / \vartheta v|<<1$. At the particle velocities considered, $b$ may be chosen such that this restriction holds for the $\omega$-values of importance. By further changing the path of integration from the positive real axis to the negative imaginary axis and back to $+\infty$ along an infinite radius, one obtains after adding $\left(-\mathrm{d} E / \mathrm{d} x_{<b}\right)$ :

$$
-\frac{\mathrm{d} E}{\mathrm{~d} x}=\frac{4 \pi q^{2} e^{4}}{m v^{2}} N Z\left[\ln 2 m v^{2}-\ln I\right]
$$

$\operatorname{In}(7) \ln I=\ln \hbar-J$

$$
J=\lim _{R \rightarrow \infty}\left[\frac{m}{4 \pi N Z e^{2}} \int_{0}^{R}\left(1-\frac{1}{\varepsilon(-i x)}\right) x \mathrm{~d} x-\ln R\right]
$$

Acta Chem. Scand. 16 (1962) No. 9 


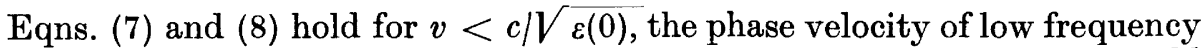
light in the medium, except for a term $\left\{-\beta^{2}-\ln \left(1-\beta^{2}\right)\right\} \cong-\frac{1}{2} \beta^{4}$ which should be added to the bracket in (7) when $\beta \leqslant 1$. When relativistic velocities are taken into account, the same term enters (4) with equal contributions from (2) and (3).

For $\varepsilon$ we should use the following form

$$
\varepsilon=1+\frac{\frac{4 \pi N Z e^{2}}{m} \sum_{i} \frac{f_{i}}{\omega_{i}^{2}-\omega^{2}+i \omega g_{i}}}{1-(1-\gamma) \frac{4 \pi N Z e^{2}}{m} \sum_{i} \frac{f_{i}}{\omega_{i}^{2}-\omega^{2}+i \omega g_{i}}}
$$

The Lorentz-Lorenz correction for the local field fixes $\gamma$ to a value of $2 / 3$. We shall, however, leave the particular values of $\gamma$ open so that its influence on the polarization effect becomes clear.

Introducing (10) into (9) gives

$$
J=\lim _{R \rightarrow \infty}\left[\int_{0}^{R} \frac{\sum_{i} \frac{f_{i}}{\omega_{i}^{2}+x^{2}+g_{i} x}}{1+\gamma \frac{4 \pi N Z e^{2}}{m} \sum_{i} \frac{f_{i}}{\omega_{i}^{2}+x^{2}+g_{i} x}} x \mathrm{~d} x-\ln R\right]
$$

In the limit of low densities ( $N$ small) we obtain

$$
J=\lim _{R \rightarrow \infty}\left[\int_{0}^{R} \sum_{i} \frac{f_{i}}{\omega_{i}^{2}+x^{2}+g_{i} x} x \mathrm{~d} x-\ln R\right]
$$

Leaving out of consideration metals and strongly polar liquids, we may neglect the $g_{i}: \mathrm{s}$ in comparison with the $\omega_{i}: \mathrm{s}$ and obtain from (12)

$$
J=\sum_{i} f_{i} \ln \omega
$$

At low densities then, (7) coincides with the Bethe formula (4) and the polarization effect is governed by the difference, $p$, between (11) and (12)

$$
\begin{gathered}
p=-\frac{1}{2} \lambda \int_{0}^{\infty} \frac{\varphi^{2}(z)}{1+\lambda \varphi(z)} \mathrm{d} z \\
\text { where } \lambda=\gamma \frac{4 N Z e^{2}}{m}, n_{i}=\omega_{i}^{2} \text {, and } \varphi(z)=\sum_{i} \frac{f_{i}}{n_{i}+z}
\end{gathered}
$$

The total energy loss becomes

$$
-\frac{\mathrm{d} E}{\mathrm{~d} x}=\frac{4 \pi q^{2} e^{4}}{m v^{2}} N Z\left[\Sigma f_{i} \ln \frac{2 m v^{2}}{\hbar \omega_{i}}+p\right]
$$

Since $p$ is negative the polarization effects a reduction in the energy loss which has also been found experimentally ${ }^{28}$.

Before undertaking the evaluation of (14) we note that the $f_{i}$ :s enter linearly into the sums. It is therefore free from the ambiguities referred to in the introduction. 


\section{EVALUATION of $p$}

$\varphi(z)$ decreases monotonously with $z$ so that the most important contribution to $p$ comes from low $z$-values. A first approximation is therefore obtained by putting

$$
\varphi(z) \cong \frac{1}{n_{\mathrm{o}}+\mathrm{z}}
$$

where

$$
\frac{1}{n_{\mathrm{o}}}=\sum_{i} \frac{\dot{j}_{i}}{n_{i}}
$$

$\varphi(z)$ and $1 /\left(n_{\mathrm{o}}+\mathrm{z}\right)$ are then equal for $z=0$. Introducing (17) into (14) gives

$$
p \cong-\frac{1}{2} \ln \frac{n_{\mathrm{o}}+\lambda}{n_{\mathrm{o}}}=-\frac{1}{2} \ln \frac{\varepsilon(0)}{\gamma+(1-\gamma) \varepsilon(0)}
$$

If $\gamma$ is put equal to $1,(19)$ agrees, as it should, with the expression obtained by Fermi ${ }^{14}$ who neglected the local field correction.

In order to investigate under which conditions (19) is a good approximation, we put

$$
\frac{1}{n_{\mathrm{i}}}=\frac{1+\delta_{i}}{n_{\mathrm{o}}}
$$

From (20) and (18) follows

$$
\sum_{i} f_{i} \delta_{i}=0
$$

and

$$
\varphi(z)=\frac{1}{n_{\mathrm{o}}+z}+\left(\sum_{i} f_{i} \delta_{i}\right) \frac{n_{\mathrm{o}}}{\left(n_{\mathrm{o}}+z\right)^{2}}-\left(\sum_{i} f_{i} \delta_{i}^{2}\right) \frac{z n_{\mathrm{o}}}{\left(n_{\mathrm{o}}+z\right)^{3}}+\cdots
$$

The second term on the right hand side of (22) is zero because of (21) and the validity of the approximation depends on whether the third and higher terms are small. If the oscillator strengths $f_{i}$ were concentrated to $1 / n_{i}$-values forming a region of small extent compared to $1 / n_{\mathrm{o}}, \Sigma f_{i} \delta_{i}^{2}$ etc. would be small and the approximation (17) justified. Since $f_{i}$ decreases rapidly with increasing $\omega_{i}$ for transitions to the continuum, it might appear reasonable to assume that when there are "narrow gaps" between the first excited level and the ionization limit this requirement would be fulfilled ${ }^{24}$. It is therefore of primary interest to get an idea of the variations of $f_{i}$ with $1 / n_{i}$ in typical atomic and molecular systems. It will be seen that $f_{i}$ as a function of $1 / n_{i}$ is not confined to a small region around $1 / n_{\mathrm{o}}$. The situation is rather the opposite, thus invalidating the approximation (17).

\subsection{Some oscillator strength distributions}

The oscillator strength for a dipole transition from the ground state of an atom or molecule containing $Z$ electrons is defined by 


$$
f_{i}=\frac{E_{i}-E_{\mathrm{o}}}{3 Z} \mid\left(0\left|\sum \vec{r}_{j}\right| i\right)^{2}
$$

$E_{\mathrm{o}}$ and $E_{i}$ are the energies of the ground and $i$ :th excited state respectively, measured in rydbergs, $\vec{r}_{j}$ is the position vector of the $j:$ th electron and

$$
\left(0\left|\vec{\sum}_{j}\right| i\right)=\int \Psi_{\circ}^{*}\left(\vec{\sum}_{j}\right) \Psi_{i} \mathrm{~d} \tau
$$

where $\Psi_{\mathrm{o}}$ and $\Psi_{i}$ are the ground and excited state wave functions. The unit of length is the Bohr radius, $\hbar^{2} /\left(m e^{2}\right)$

Eqn. (24) has been calculated exactly for all transitions of the hydrogen atom including the continuum ${ }^{29,30}$. For systems with more than one electron, the exact wave functions are not known and approximate ones have to be used. Since the matrix element (24) depends on the overlap between the ground and excited state wave functions, it is very sensitive to errors in these functions. The approximate wave functions are generally developed to give accurate expectation values of the energy. Since the latter is stationary with respect to variations of the wave function, large errors in the matrix elements (24) may result. Fairly accurate calculations exist, however, for the helium atom and the negative hydrogen ion based on Hylleraas wave functions for the ground state and suitable approximations for the excited state ${ }^{31,32}$.

For other systems, values have been computed only for individual transitions from which it is difficult to obtain an overall picture.

Experimental determination of oscillator strengths is possible by several methods, e.g. light absorption measurements. A major part of these measurements has to be carried out in the vacuum ultraviolet region where large experimental difficulties appear ${ }^{33}$. Recently measurements have been reported for $\mathrm{H}_{2}$ and $\mathrm{C}_{6} \mathrm{H}_{6}$ covering a large part of the continuum past the ionization limits $^{34}$.

3.11 The hydrogen atom. For the discrete spectrum we have ${ }^{35}$

$$
E_{n}-E_{\mathrm{o}}=1-\left(1 / n^{2}\right)
$$

and

$$
|(0|x| n)|^{2}=\frac{2^{8}}{3} n^{7} \frac{(n-1)^{2 n-5}}{(n+1)^{2 n+5}}
$$

where $n$ is the principal quantum number. The $n$ :th level is $n^{2}$ fold degenerate but the matrix elements of $x$ are zero except for $n p_{x}$ states and similarly for $y$ and $z$ so that

$$
\frac{1}{3}|(0|\vec{r}| n)|^{2}=|(0|x| n)|^{2}
$$

Thus

$$
f_{n}=\frac{2^{8}}{3} n^{5} \frac{(n-1)^{2 n-4}}{(n+1)^{2 n+4}}
$$


In the continuum $n$ is pure imaginary and may be written

$$
n=1 / i k
$$

where $i$ is the imaginary unit and $2 \pi / k$ is the wave length of the electron at an infinite distance from the nucleus. With the wave function for the excited state normalized with regard to $k$, i.e.

$$
\int \Psi_{k^{\prime}} \Psi_{k} \mathrm{~d} \tau=\delta\left(k^{\prime}-k\right),
$$

the absolute square of the matrix element is ${ }^{35}$

$$
|(0|x| n)|^{2}=-\frac{2^{8}}{3} \frac{|n|^{9}}{1-\mathrm{e}^{-2 \pi|n|}} \frac{(n-1)^{2 n-5}}{(n+1)^{2 n+5}}
$$

and

$$
f_{n}=\frac{2^{8}}{3} \cdot \frac{|n|^{7}}{1-\mathrm{e}^{-2 \pi|n|}} \frac{(n-1)^{2 n-4}}{(n+1)^{2 n+4}}
$$

As we wish to plot $f$ as a function of $\eta=1 / \omega_{i}^{2}$, it must be normalized with respect to the same variable, i.e.

$$
f(\eta)|\mathrm{d} n|=f_{n}|\mathrm{~d} k|
$$

Since

$$
\begin{aligned}
\eta & =1 /\left[1-\left(1 / n^{2}\right)\right]^{2} \\
f(\eta) & =\frac{2^{6}}{3} \cdot \frac{|n|^{2}}{1-\mathrm{e}^{-2 \pi|n|}} \frac{(n-1)^{2 n-1}}{(n+1)^{2 n+1}}
\end{aligned}
$$

or expressed in the real variable $k$

and

$$
\eta=\left[k^{2} /\left(1+k^{2}\right)\right]^{2}
$$

$$
f(\eta)=\frac{2^{6}}{3} \frac{\exp [-(4 / k) \operatorname{arctg} k]}{\left(1+k^{2}\right)[1-\exp (-2 / k)]}
$$

$f(\eta)$ is shown diagramatically in Fig. la where the oscillator strengths for the discrete transitions have been spread out into block form. $f(\eta)$ is of a remarkably simple form extending with little change all the way down to zero abscissa. For the full curve drawn in this and some of the other figures, see section 3.2 .

3.12 Helium and the negative hydrogen ion. Available theoretical values of the oscillator strengths of helium for both the discrete and continuous spectrum have been critically reviewed by Dalgarno and Lynn ${ }^{31}$ and modified so as to agree with various experimental data. Their results are shown in Fig. $1 \mathrm{~b}$ after suitable renormalization. It has the same general appearance as for the hydrogen atom but there is an even stronger accumulation towards low $\eta$-values.

For the continuum of the $\mathrm{H}^{-}$ion, calculations have been reported by Chandrasekhar ${ }^{32}$ who gives the photoelectric cross section $\varkappa_{v}$ from zero wave length to $16550 \AA$, the ionization limit. In the same units as above

Acta Chem. Scand. 16 (1962) No. 9 


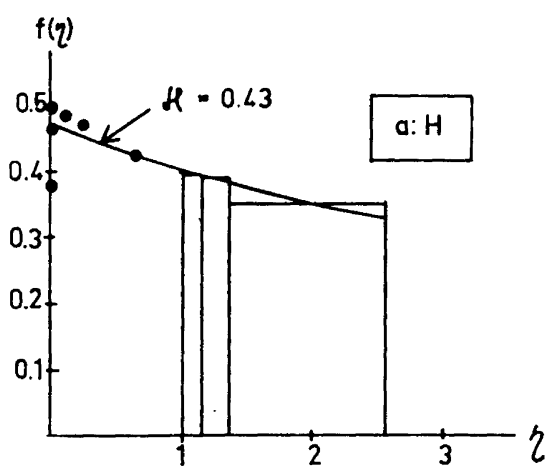

$f(\eta)$
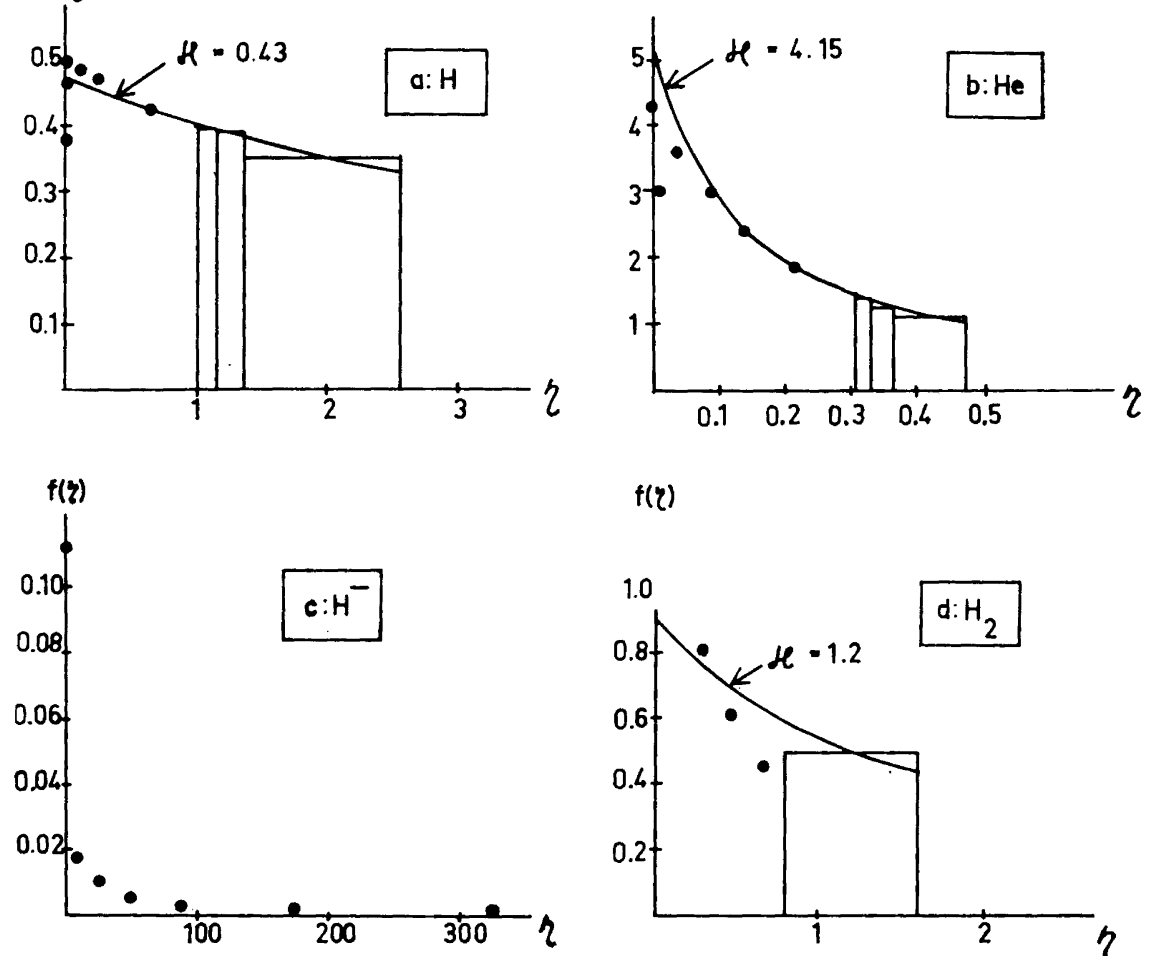

$f(\eta)$
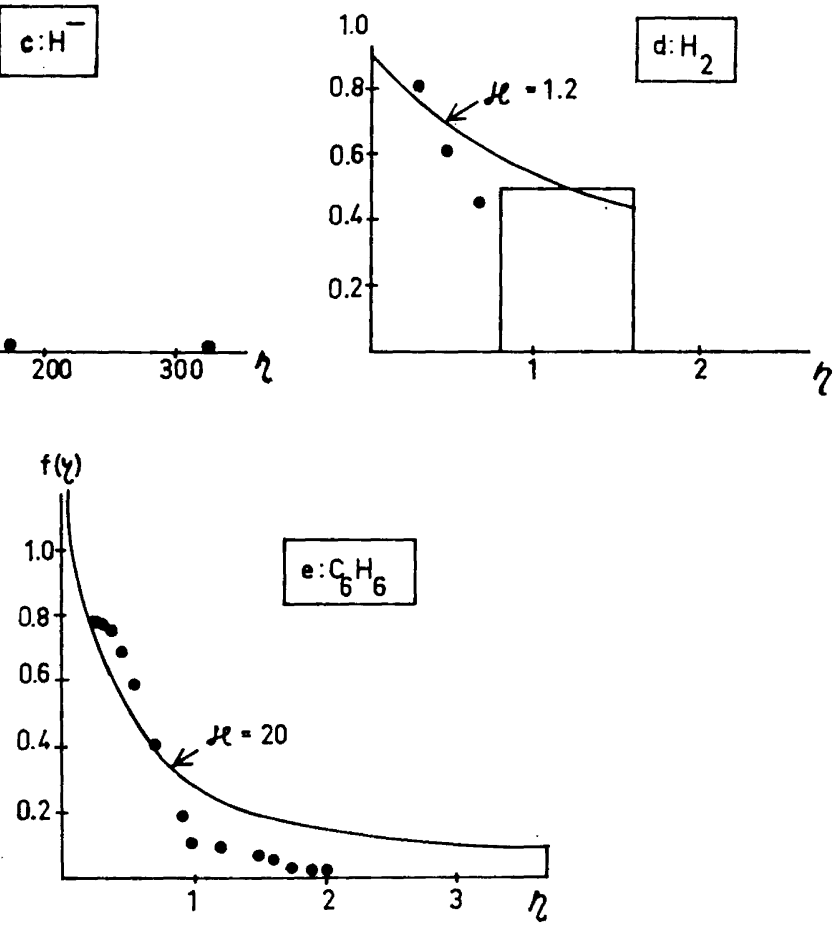

Fig. $1 a-e$. Oscillator strength distributions as functions of $\eta=1 / \omega^{2}$, the latter in units of the inverse square of the Rydberg frequency: $m e^{4} / 2 \hbar^{3}$. Calculated or measured values are indicated by dots in the continuum and by blocks for "discrete" transitions. Figs. d and, in particular, e are incomplete. The full curves are approximations of the form given in equation (41). 


$$
f(\eta)=23.5 \varkappa_{v} / \lambda^{3}
$$

and

$$
\eta=1.21 \times 10^{10} \lambda^{2}
$$

where $\varkappa_{\nu}$ is given in $\mathrm{cm}^{2}$ and $\lambda$ in $\mathrm{cm}$. The results are shown in Fig. 1c and are found to be drastically different from the previous ones. The oscillator strength tends to zero at the ionization limit, a behaviour entirely different from that of $\mathrm{H}$ and $\mathrm{He}$. It is due to the circumstance that the excited electron in the $\mathrm{H}^{-}$ ion moves effectively in the field from an electrically neutral residue whereas for $\mathrm{H}$ and $\mathrm{He}$ this residue gives a coulomb field at large distances ${ }^{36}$. The tendency towards an accumulation of oscillator strength at low $\eta$-values is here extreme.

3.13 Hydrogen and benzene. From the absorption constant, $\mu$, given by Bunch et al. ${ }^{34}$, one obtains

$$
f(\eta)=1.75 \times 10^{12} \mu / \lambda^{3}
$$

with $\eta$ given by (36). It is plotted in Figs. 1d and e. The oscillator strengths for the transitions to electronic levels below the ionization limits were not measured. For $\mathrm{H}_{2}$ we have added a block of the content 0.42 being an estimate ${ }^{35}$ of the sum of the oscillator strengths of all transitions below the ionization limit. At $\eta$-values somewhat smaller than the ionization limit, there should be contributions from doubly excited levels so that the overall shape becomes again similar to that of $\mathrm{H}$ and $\mathrm{He}$.

For benzene the data are even more incomplete. No transitions to states below the ionization limits are included. Corresponding to the different ionization energies of the various $\pi$ and $\sigma$ electrons, there appear several steps. These will probably be largely filled out in a diagram containing all transitions. At $\eta \sim 0.002$, there will be a contribution from the $\mathrm{K}$ shell electrons of the carbon atoms. The important feature is again the monotonous decrease of $f$ versus $\eta$.

\subsection{A two-parameter description of $f(\eta)$}

To take into account the features of $f(\eta)$ which were found above, we shall approximate $f\left(r_{l}\right)$ by means of the following continuous expression

$$
\begin{aligned}
& f(\eta)=\frac{a}{b+\eta} \quad 0<\eta<\eta_{m} \\
& f(\eta)=0 \quad \eta>\eta_{m}
\end{aligned}
$$

The requirement that $\int f(\eta) \mathrm{d} \eta=1$ reduces the number of independent parameters to two.

$$
\int f(\eta) \mathrm{d} \eta=a \ln \left[1+\left(\eta_{m} / b\right)\right]=1
$$

Introducing

$$
\eta_{m} / b=x
$$


(38) reads

$$
\begin{aligned}
f(\eta) & =\frac{1}{\ln (1+x)} \cdot \frac{1}{\mathrm{~b}+\eta} & 0<\eta<\eta_{m} \\
f(\eta) & =0 & \eta>\eta_{m}
\end{aligned}
$$

$\varkappa$ determines the form, $\eta_{m}$ the scale, of $f(\eta)$.

Inserting (41) into (15) we obtain the following expression for $\varphi(z)$ :

$$
\varphi(z)=\int_{0}^{\infty} \frac{f(\eta)}{(1 / \eta)+z} \mathrm{~d} \eta=\eta_{m} F(x)
$$

where $x=\eta_{m} z, \beta=(1 / x) \ln (1+x)$, and

$$
F(x)=\frac{\beta x-\ln (1+x)}{\beta x(x-x)}
$$
for $p$ :

Changing to $x$ as the integration variable we obtain the following expression

$$
p=-\frac{1}{2} \tau \int_{0}^{\infty} \frac{F^{2}(x)}{1+\tau F(x)} \mathrm{d} x ; \quad \tau=\lambda \eta_{m}
$$

An exact evaluation of (44) would probably yield a quite unwieldy expression. We shall develop two approximate expressions for $p$, viz.

and

$$
p=-\frac{1}{2} \tau \int_{0}^{\infty} \frac{F_{1}^{2}(x)}{1+\tau F_{1}(x)} \mathrm{d} x
$$

$$
p=-\frac{1}{2} \tau \int_{0}^{\infty} F^{2}(x) \mathrm{d} x+\frac{1}{2} \tau^{2} \int_{0}^{\infty} \frac{F_{1}^{3}(x)}{1+\tau F_{1}(x)} \mathrm{d} x
$$

where

$$
F_{1}(x)=\frac{F(0)}{1+\alpha x}
$$

which coincides with $F(x)$ at $x=0$. With $F$ in place of $F_{1},(46)$ is identical to (44). Choosing $\alpha$ such that

$$
F_{1}^{\prime}(0)=F^{\prime}(0)
$$

we obtain an approximation which is accurate for small $x$-values, i.e. where $F$ is large and gives the essential contribution to the integral in (45) and the last integral in (46).

Eqn. (46) will be a more accurate approximation than (45) since the main contribution from $F_{1}^{3}(x)$ is even more concentrated to low $x$-values than that from $F_{1}^{2}(x)$.

One finds

$$
\alpha=\frac{1}{1-\beta}\left(\frac{1}{2}-\frac{1-\beta}{x}\right)
$$

Acta Chem. Scand. 16 (1962) No. 9 
Eqns. (45) and (46) become

$$
p=-\frac{1}{2} \frac{F(0)}{\alpha} \ln (1+\tau F(0))
$$

and

$$
p=-\frac{1}{2} \tau F(0) \cdot G(\varkappa)+\frac{1}{2} \frac{F(0)}{\alpha}[\tau F(0)-\ln (1+\tau F(0))]
$$

where *

$$
\begin{gathered}
G(x)=\frac{\beta x}{1-\beta}\left[-\frac{\ln (1+x)}{3 x}-\frac{1}{x(1+x)}+\frac{4 \zeta(2)}{\ln ^{2}(1+x)}\left(\frac{1+(x / 2)}{1+x}-\frac{\ln (1+x)}{x}\right)+\right. \\
\left.+2 \frac{\mathrm{L}_{2}(x /[1+x])}{\ln ^{2}(1+x)}\left(\frac{1}{1+x}-\frac{\ln (1+x)}{x}+2 \frac{\mathrm{L}_{3}(x /[1+x])}{x \ln ^{2}(1+x)}\right)\right]
\end{gathered}
$$

a rather cumbersome expression. $\zeta$ is Riemann's zeta function ${ }^{38}, \mathrm{~L}_{2}$ is Euler's dilogarithm ${ }^{39,40}$ and

$$
\mathrm{L}_{3}(z)=\sum_{j=0}^{\infty} \frac{\mathrm{B}_{j}}{j !} \frac{[-\ln (1-z)]^{j+2}}{j+2}
$$

where $\mathrm{B}_{j}$ are the Bermoulli numbers. The difference between $\left(46^{\prime}\right)$ and $\left(45^{\prime}\right)$ is $* *$

$$
\frac{1}{2} \tau F(0)\left(\frac{F(0)}{\alpha}-G(x)\right)
$$

From (10), (15), (42), and (44) we obtain

Finally

$$
1+\tau F(0)=\frac{\varepsilon(0)}{1+(1-\gamma)(\varepsilon(0)-1)}
$$

$$
\frac{F(0)}{\alpha}=\frac{(1-\beta)^{2}}{\beta \varkappa\left(\frac{1}{2}-\frac{1-\beta}{\varkappa}\right)}
$$

To calculate the polarization effect, we need $\varepsilon(0), \gamma$, and $\varkappa . \varepsilon(0)$ is usually readily available and $\gamma$ is equal to $2 / 3$ when the Clausius-Mossotti formula holds. Otherwise one has to use the $\gamma$-value giving the best agreement between (10) and experimental dispersion relations. It therefore remains to determine $x$, the form factor of the oscillator strength distribution.

\subsection{Determination of $x$}

The two independent parameters in our description of $f(\eta)$ may be taken to be $\eta_{m}$ and $\varkappa$. (53) gives one relation between these parameters since $\tau$ contains

\footnotetext{
* The author gratefully acknowledges the aid of Dr. B. Nagel in evaluating $\int_{0}^{\infty} F^{2} \mathrm{~d} x$ in terms of known transcendental functions.
}

** A further improvement is probably obtained by choosing $\alpha$ such that (52) becomes zero. 
$\eta_{m}$. A second relation is obtained from the mean excitation potential, $I_{\mathrm{o}}$, obtainable from measurements on the substance in gaseous form:

$$
\begin{gathered}
\ln \left(I_{\mathrm{o}} / \hbar\right)=\sum_{i} f_{i} \ln \omega_{i} \\
=-\frac{1}{2} \int f(\eta) \ln \eta \mathrm{d} \eta \\
=-\frac{1}{2} \ln \left(\frac{\eta_{m}}{1+x}\right)+\frac{1}{2} \frac{\mathrm{L}\left({ }_{2} x /[1+x]\right)}{\ln (1+x)}
\end{gathered}
$$

After elimination of $\eta_{m}$ between (55) and (53), there results the following relation determining $x$ :

where

$$
H(\varkappa)=h[\varepsilon(0)] \cdot\left(I_{\mathrm{o}} / \hbar\right)^{2}
$$

$$
H(x)=\left(\frac{1}{\ln (1+x)}-\frac{1}{x}\right)(1+x) \exp \frac{\mathrm{L}_{2}(x /[1+x])}{\ln (1+x)}
$$

and

$$
h[\varepsilon(0)]=\frac{m}{4 \pi N Z e^{2}} \cdot \frac{\varepsilon(0)-1}{1+(1-\gamma)(\varepsilon(0)-1)}
$$

$h(\varepsilon(0))$ is proportional to the molecular polarizability.

\section{CONCLUSIONS}

The expressions for the polarization effect arrived at here are fairly similar to that deduced by Fermi ${ }^{14}$ using a single frequency description of the dielectric constant:

$$
p=-\frac{1}{2} \ln \varepsilon(0)
$$

Our expression $\left(45^{\prime}\right)$ differs from Fermi's by the factor $F(0) / \alpha$ and the inclusion of the local field correction through $\gamma . F(0) / \alpha$ is a rather slowly varying function of $x$, being 0.750 for $x=0$ and 0.565 for $x=10$. This indicates that $p$ is fairly insensitive to the finer details in the oscillator strength distribution. In the better approximation $\left(46^{\prime}\right)$, there enters moreover the additive term (52) which is negative, reducing the variation with $\varkappa$ still further.

To test the theory, measurements should be made on substances in gaseous and liquid form. The velocity of the particles, while fulfilling (1), should be as small as possible, in order that the relative effect of $p$ in (16) should be as large as possible. For this purpose $\alpha$-particles with an energy of about $5 \mathrm{MeV}$ would be very suitable. Measurements of the energy loss of such particles in liquids may now be made with an accuracy of about $0.1 \%{ }^{41}$. A similar accuracy should be attainable in measurements on gases, thus allowing a detailed check to be made. 


\section{REFERENCES}

1. Thomson, J. J. Conduction of Electricity through Gases. Cambridge 1906, pp. 370-389.

2. Darwin, C. G. Phil. Mag. 23 (1912) 907.

3. Bohr, N. Phil. Mag. 25 (1913) 10.

4. Bohr, N. Phil. Mag. 30 (1915) 581.

5. Henderson, G. H. Phil. Mag. 44 (1922) 680.

6. Fermi, E. Z. Physik 29 (1924) 315.

7. Bethe, H. A. Ann. Physik 5 (1930) 325.

8. Bethe, H. A. Z. Physik 76 (1932) 293.

9. Bloch, F. Ann. Physik 16 (1933) 285.

10. Bloch, F. Z. Physik 81 (1933) 363.

11. Swann, W. G. F. J. Franklin Inst. 226 (1938) 598.

12. Fermi, E. Phys. Rev. 56 (1939) 1242.

13. Fermi, E. Phys. Rev. 57 (1940) 485.

14. Halpern, O. and Hall, H. Phys. Rev, 57 (1940) 459.

15. Wick, G. C. Nuovo cimento 1 (1943) 302 .

16. Halpern, O. and Hall, H. Phys. Rev. 73 (1948) 477.

17. Bohr, A. Kgl. Danske Videnskab Selskab. Matem.-fys. Medd. 24 (1948) No. 19.

18. Messel, H. and Ritson, D. Phil. Mag. 41 (1950) 1129.

19. Schoenberg, M. Nuovo cimento 8 (1951) 159.

20. Schoenberg, M. Nuovo cimento 9 (1952) 372.

21. Huybrechts, M. and Schoenberg, M. Nuovo cimento 9 (1952) 764.

22. Sternheimer, R. M. Phys. Rev. 88 (1952) 851.

23. Sternheimer, R. M. Phys. Rev. 93 (1954) 351.

24. Brandt, W. Phys. Rev. 104 (1956) 691.

25. Brandt, W. Research report, E. I. du Pont de Nemours \& Co., 1957.

26. Aniansson, G. Trans. Roy. Inst. Techn. 1961 No. 178.

27. Mott, N. F. Proc. Gambridge Phil. Soc. 27 (1931) 553.

28. Aniansson, G. Phys. Rev. 98 (1955) 500.

29. Sugiura, Y. Sci. Papers Inst. Phys. Chem. Res. 11 (1929) 1.

30. Gordon, W. Ann. Physik 2 (1929) 1031.

31. Dalgarno, A. and Lynn, N. Proc. Phys. Soc. A 70 (1957) 802.

32. Chandrasekhar, S. and Elbert, D. D. Astrophys. J. 128 (1958) 633.

33. Price, W. C. Advances in Spectroscopy 1 (1959) 56.

34. Bunch, S. M., Cook, G. R., Ogawa, M. and Ehler, A. W. J. Chem. Phys. 28 (1958) 740.

35. Sommerfeld, A. Wellenmechanik, Braunschweig 1939.

36. Bethe, H. A. and Salpeter, E. E. Encyclopedia of Physics, Vol. 35, p. 402. Berlin 1957.

37. Mulliken, R. S. and Rieke, C. A. Repts. Progr. Phys. 8 (1941) 231.

38. Jahnke, E. and Emck, F. Tables of Functions, Leipzig 1933, p. 319.

39. Mitchell, K. Phil. Mag. 40 (1949) 351.

40. Erdélyi, A. et al. Higher Transcendental Functions, Vol. I, p. 31, New York 1953.

41. Aniansson, G. Trans. Roy. Inst. Techn. 1961 No. 177.

Received April 14, 1962. 